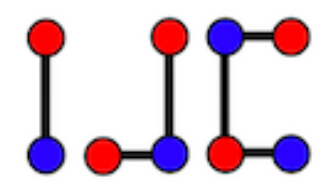

\title{
Broader families of cordial graphs
}

\author{
Christian Barrientos ${ }^{\mathrm{a}}$, Sarah Minion ${ }^{\mathrm{b}}$ \\ ${ }^{a}$ Valencia College, Orlando, FL 32832, U.S.A \\ ${ }^{b}$ Full Sail University, Winter Park, FL 32792, U.S.A \\ chr_barrientos@yahoo.com, sarah.m.minion@gmail.com
}

\begin{abstract}
A binary labeling of the vertices of a graph $G$ is cordial if the number of vertices labeled 0 and the number of vertices labeled 1 differ by at most 1 , and the number of edges of weight 0 and the number of edges of weight 1 differ by at most 1 . In this paper we present general results involving the cordiality of graphs that results of some well-known operations such as the join, the corona, the one-point union, the splitting graph, and the super subdivision. In addition we show a family of cordial circulant graphs.
\end{abstract}

Keywords: cordial, corona, join, splitting, circulant, super subdivision Mathematics Subject Classification: 05C78

DOI: $10.19184 /$ ijc.2021.5.1.6

\section{Introduction}

All graphs considered here are finite with no loops nor multiple edges. The notation used follows the one used in [4] and [6]. By a $(m, n)$ graph we mean a graph of order $m$ and size $n$. In this paper, a binary labeling of a graph $G$ is a function that assigns to each vertex of $G$ one of the two labels 0 or 1, where every edge $u v$ of $G$ has assigned a weight defined by $|f(u)-f(v)|$. If $f$ is a binary labeling of a graph $G$, for $x \in\{0,1\}, v_{f}(x)$ and $e_{f}(x)$ denote the number of vertices labeled $x$ by $f$ and the number of edges of weight $x$, respectively.

A graph $G$ is cordial if there exists a binary labeling $f$ of $G$ such that $\left|v_{f}(0)-v_{f}(1)\right| \leq 1$ and $\left|e_{f}(0)-e_{f}(1)\right| \leq 1$. A cordial labeling of a graph can be represented as a coloring of the vertices and edges of the graph, where the vertices labeled 0 are colored in black while the ones labeled

Received: 10 November 2020, Revised: 24 January 2021, Accepted: 10 February 2021. 
1 are colored white, and the edges of weight 0 are represented by solid lines, while the ones of weight 1 correspond to dashed lines. On all the figures in this work, we just use the coloring of the vertices to represent the cordial labelings.

There is a long list of families of cordial graphs, a detailed account of them can be found in [6]. Among the most general results we can find the following: the maximal number of edges in a cordial graph is known [5]; if $G$ is a $(m, n)$ graph such that every vertex has odd degree, then $G$ is not cordial when $m+n \equiv 2(\bmod 4)$ [12], every Eulerian $(m, n)$ graph is not cordial when $n \equiv 2(\bmod 4)[2]$; all unicyclic graphs are cordial except for $C_{n}$ when $n \equiv 2(\bmod 4)$ [8]; a cubic graph of order $m$ is cordial if and only if $m \not \equiv 4(\bmod 8)[10]$; every Skolem-graceful graph is cordial [15]; graceful and harmonious labelings of trees can be transformed into cordial labelings [2]. Several operations involving cordial graphs have been studied: the Cartesian product of two cordial graphs of even size is cordial, the composition of two cordial graphs is cordial if one of them has odd order and even size [8]; the union of two cordial graphs is cordial if one of them has even size, the join of two cordial graphs is cordial when both graphs have even size and either one has even order [16].

In this paper we present several general results. In Section 2 we analyze cordial labelings of graphs that result of the corona of two cordial graphs. In Section 3 we present a cordial labeling of some circulant graphs; in addition, we prove that all splitting graphs of a cordial graph of even order and even size, is cordial. In Section 4 we focus on the cordiality of graphs that result of the super subdivision of the edges of some graphs. In Section 5 we study the cordiality of the join of two cordial graphs. In Section 6 we consider the cordiality of the one-point union of $t$ copies of a cordial graph.

\section{Cordial Corona Graphs}

Let $f$ be a cordial labeling of a graph $G$. The complementary labeling of $f$, denoted by $\bar{f}$, is defined for every $v \in V(G)$ as $\bar{f}(v)=1-f(v)$. Note that $\bar{f}$ is also cordial and preserves the weights. Thus, we may assume that for every cordial graph $G$ of odd order, there is a cordial labeling $f$ such that $v_{f}(0)-v_{f}(1)=1$.

For $i \in\{1,2\}$, let $G_{i}$ be a $\left(m_{i}, n_{i}\right)$ graph. The corona $G_{1} \odot G_{2}$ is the graph obtained by taking one copy of $G_{1}$ and $m_{1}$ copies of $G_{2}$ and then joining the $i$ th vertex of $G_{1}$ to all the vertices in the $i$ th copy of $G_{2}$. In general, this operation is not commutative. In [1], Andar et al. studied cordial labelings of graphs of the form $G \odot n K_{1}$. In this section we consider a related, but different, problem. Let $G$ be a $(m, n)$ cordial graph, is $K_{1} \odot G$ cordial? In order to answer this question, we analyze four cases. Suppose that $g$ is a cordial labeling of $G$ and that $w$ is the vertex of $K_{1}$.

Case 1. When $m$ and $n$ are even.

The cordial labeling $g$ of $G$ satisfies $v_{g}(0)-v_{g}(1)=0$ and $e_{g}(0)-e_{g}(1)=0$. We can extend $g$ to a cordial labeling $f$ of $K_{1} \odot G$ by assigning, for instance, the label 0 to $w$, that is, $f(w)=0$. Thus, $v_{f}(0)-v_{f}(1)=1$. Since $v_{g}(0)-v_{g}(1)=0$, exactly half of the edges between $K_{1}$ and $G$ have weight 0 , i.e., $e_{f}(0)-e_{f}(1)=0$ and $K_{1} \odot G$ is cordial. Recall that $K_{1} \odot G=G+K_{1}$. Thus, this case, as well as Case 3, are just specific instances of the result of Youssef [15], where he 
proves that if $G$ and $H$ are cordial and both have even size, then $G+H$ is cordial.

Case 2. When $m$ is odd and $n$ is even.

In this case we use $f(w)=1$ when $v_{g}(0)-v_{g}(1)=1$ and $f(w)=0$ when $v_{g}(0)-v_{g}(1)=-1$. Either way, the number of edges of weight 1 , between $K_{1}$ and $G$, is $\frac{m+1}{2}$. So, $v_{f}(0)-v_{f}(1)=0$ and $e_{f}(0)-e_{f}(1)=-1$; consequently, $f$ is cordial.

Case 3. When $m$ is even and $n$ is odd.

Regardless of the difference between $e_{g}(0)$ and $e_{g}(1)$, the vertex $w$ can be labeled either 0 or 1 to obtain a cordial labeling of $K_{1} \odot G$, because exactly half of the edges between $K_{1}$ and $G$ have weight 0 . Then $\left|v_{f}(0)-v_{f}(1)\right|=1$ and $\left|e_{f}(0)-e_{f}(1)\right|=1$.

Case 4. When $m$ and $n$ are odd.

Recall that in this case we can assume the existence of a cordial labeling $g$ of $G$ such that $v_{g}(0)-v_{g}(1)=1$. If $e_{g}(0)-e_{g}(1)=1$, then $f(w)=1$. Thus, $v_{f}(0)-v_{f}(1)=0$ and exactly $\frac{m+1}{2}$ of the edges between $K_{1}$ and $G$ have weight 1 . Therefore $e_{f}(0)-e_{f}(1)=0$ and $f$ is cordial. If $e_{g}(0)-e_{g}(1)=-1$, then $f(w)=1$, because $v_{g}(0)-v_{g}(1)=1$. Thus, exactly $\frac{m+1}{2}$ of the edges between $K_{1}$ and $G$ have weight 1 and $f$ is not cordial because $e_{f}(0)-e_{f}(1)=-2$. Is this last instance possible? That is, does there exist a cordial graph of odd order and odd size such that all its cordial labelings induce more edges of weight 1 than edges of weight 0 ? Note that if we use a labeling $g^{\prime}$ of $G$ such that $v_{g^{\prime}}(0)-v_{g^{\prime}}(1)=-1$, then we will encounter the same problem.

Let $\mathscr{O}$ be the family of all cordial graphs of odd order and odd size for which there is no cordial labeling $g$ such that $e_{g}(0)-e_{g}(1)=1$. In other terms, if $G \in \mathscr{O}$, then any cordial labeling $g$ of $G$ satisfies $e_{g}(0)-e_{g}(1)=-1$. We know that for every $n \equiv 3(\bmod 4)$, the cycle $C_{n}$ is a member of $\mathscr{O}$. Therefore, the wheel $W_{n}=K_{1} \odot C_{n}$ is not cordial when $n \equiv 3(\bmod 4)$.

After an exhaustive search that included all 1043 graphs of order up to seven with no isolated vertices [11], we know that only 23 of these graphs are members of $\mathscr{O}$, that is, all their cordial labelings have an extra edge of weight 1 . We show them in Figure 1, the vertex coloring exhibited, corresponds to a cordial labeling. So we may conclude that most of the graphs of the form $K_{1} \odot G$ are cordial provided that $G$ is cordial. Using this notation and the calculations done before, we can establish the following theorem.

Theorem 2.1. If $G$ is a cordial graph such that $G \notin \mathscr{O}$, then the corona $K_{1} \odot G$ is cordial.

This result can be used to prove the cordiality of many families of related graphs.

Theorem 2.2. Let $G$ and $H$ be cordial graphs. If $G$ has even order and even size, then the corona $H \odot G$ is cordial.

Proof. Let $g$ and $h$ be cordial labelings of $G$ and $H$, respectively. Since $G$ has even order and even size, we have that $v_{g}(0)-v_{g}(1)=0$ and $e_{g}(0)-e_{g}(1)=0$. By Theorem 2.1, we know that there exists a labeling $f$ of $K_{1} \odot G$ such that $e_{f}(0)-e_{f}(1)=0$ and $v_{f}(0)-v_{f}(1)=1$ or $v_{f}(0)-v_{f}(1)=-1$. Let $H$ be a $(m, n)$ graph. Since $H \odot G$ can be seen as the graph formed by $m$ copies of $K_{1} \odot G$, where the vertices $w$ on the copies of $K_{1} \odot G$ are connected following the edges 


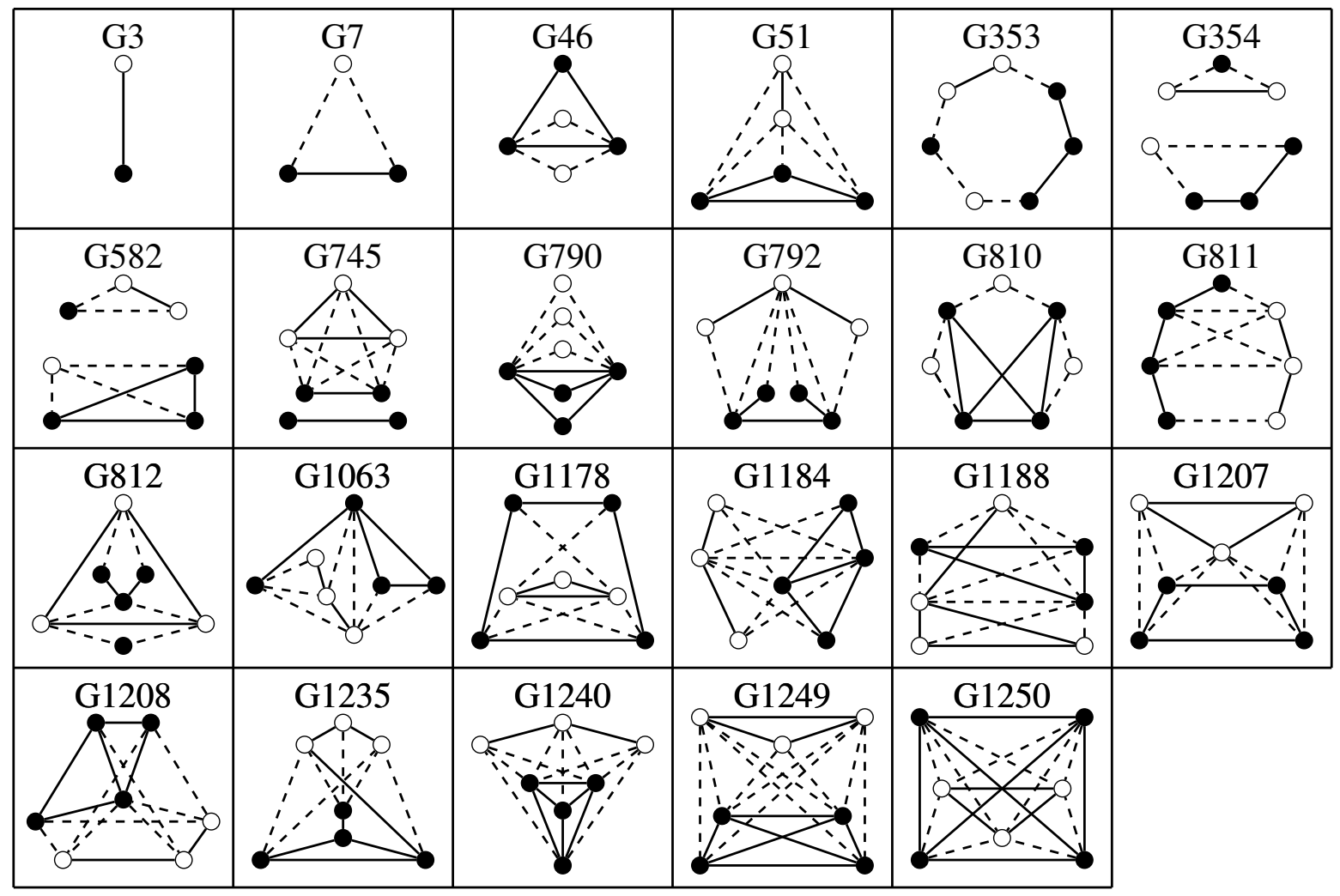

Figure 1. Smallest members of $\mathscr{O}$

of $H$, a cordial labeling of $H \odot G$ is obtained using $v_{h}(0)$ copies of $K_{1} \odot G$ where the vertices $w$ are labeled 0 and $v_{h}(1)$ copies of $K_{1} \odot G$ where the vertices $w$ are labeled 1 . The resulting labeling fof $H \odot G$ satisfies $v_{f}(0)-v_{f}(1)=v_{h}(0)-v_{h}(1)$ and $e_{f}(0)-e_{f}(1)=e_{h}(0)-e_{h}(1)$. Since $h$ is cordial, $f$ is also cordial.

Many families of cordial graphs are obtained in this form. For instance: $P_{m} \odot C_{4 k}$ for all positive integers $m$ and $k ; C_{m} \odot C_{4 k}$ for all positive integers $m$ and $k$ where $m \not \equiv 2(\bmod 4)$. In Figure 2 we show an example where $H \cong C_{5}$ and $C_{4 k} \cong C_{4}$.

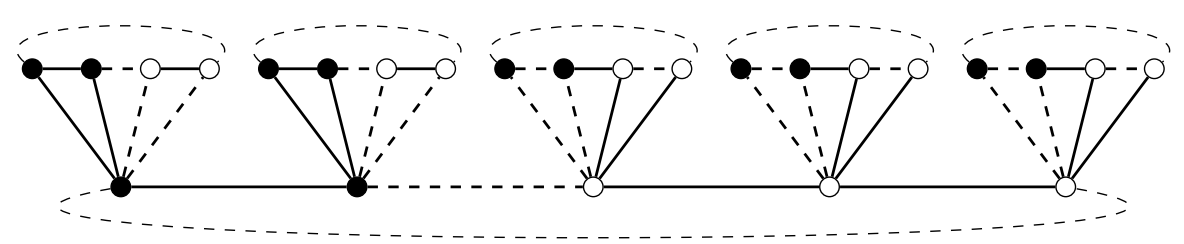

Figure 2. Cordial labeling of the corona $C_{5} \odot C_{4}$

Theorem 2.3. Let $G$ and $H$ be cordial graphs. If $G$ has odd order, odd size, and $G \notin \mathscr{O}$, then $H \odot G$ is cordial. 
In this case, the labeling $f$ of the copies of $K_{1} \odot G$, obtained using Theorem 2.1, satisfies $v_{f}(0)-v_{f}(1)=0$ and $e_{f}(0)-e_{f}(1)=0$. Thus, Theorem 2.3 can be proved using the argument employed in Theorem 2.2, so we omit the proof. However, we show an example of the labeling obtained in this case where $H \cong S_{1,4} \cong K$ and $G \cong C_{5}$.

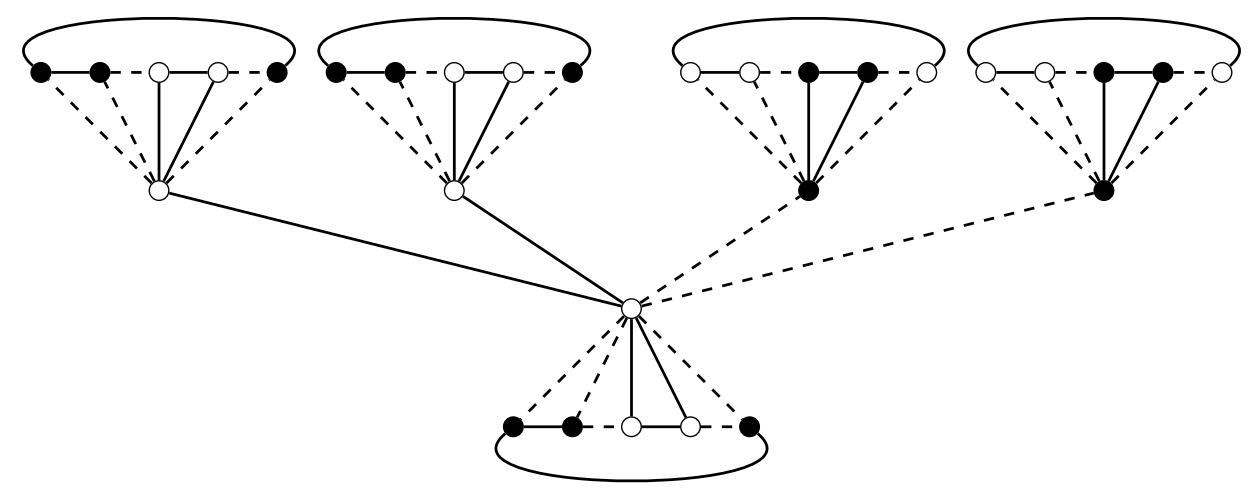

Figure 3. Cordial labeling of the corona $S_{4} \odot C_{5}$

Theorem 2.4. Let $G$ be a cordial graph of odd order and even size. If $H$ is a $(m, n)$ graph, where $n \in\{m-1, m, m+1\}$, then the corona $H \odot G$ is cordial.

Proof. Recall that in this case, there exists a cordial labeling $f$ of $K_{1} \odot G$ such that $v_{f}(0)-v_{f}(1)=$ 0 and $e_{f}(0)-e_{f}(1)=-1$. Without loss of generality, we assume that the vertex $w$ of every copy of $K_{1} \odot G$ is labeled 0 by $f$. Thus, when every copy of $H \odot G$ has been labeled, there are $m$ more edges of weight 1 , but all the $n$ edges of $H$ have weight 0 . Since $n \in\{m-1, m, m+1\}$, $|m-n| \leq 1$ and $H \odot G$ is cordial.

In Figure 4 we show an example of this result, where $H \cong C_{3}$ and $G \cong P_{5}$.

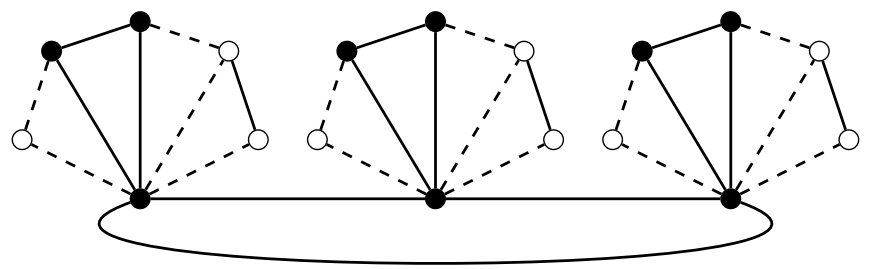

Figure 4. Cordial labeling of the corona $C_{3} \odot P_{5}$

Let $G$ be a cordial $(m, n)$ graph. From Theorem 2.1 we know that when $m$ is even and $n$ is odd, there exists a cordial labeling $f$ of $K_{1} \odot G$ such that $\left|v_{f}(0)-v_{f}(1)\right|=1$ and $\left|e_{f}(0)-e_{f}(1)\right|=1$. Thus, if the cordial labeling $g$ of $G$ satisfies $e_{g}(0)-e_{g}(1)=-1$ and the vertex $w$ of $K_{1} \odot G$ is labeled 0 by $f$, we can prove a result similar to the one on Theorem 2.4. A more interesting case appears when $e_{g}(0)-e_{g}(1)=1$. 
We say that a bipartite graph $H$ is balanced when the absolute difference of the cardinalities of the partite sets of $V(H)$ is at most one. In the next theorem we prove that under these conditions, the corona $H \odot G$ is cordial for some cordial graphs of even order and odd size.

Theorem 2.5. Let $H$ be a balanced $(m, n)$ graph and $G$ be a cordial graph of even order and odd size. If $g$ is a cordial labeling of $G$ such that $e_{g}(0)-e_{g}(1)=1$, then the corona $H \odot G$ is cordial.

Proof. Since $H$ is balanced, $n \in\{m-1, m, m+1\}$. Suppose $\{B, W\}$ is the bipartition of $V(H)$; without loss of generality, we assume $|B| \geq|W|$. Let $h: V(H) \rightarrow\{0,1\}$ defined by

$$
h(v)= \begin{cases}0 & \text { if } v \in B \\ 1 & \text { if } v \in W .\end{cases}
$$

Thus, $e_{h}(1)-e_{h}(0)=n$ and $v_{h}(0)-v_{h}(1) \leq 1$.

The cordial labeling $f$ of each $K_{1} \odot G$ is taken in such a way that $f(w)$ matches with the label of the vertex of $H$ associated to it. Thus, the number of vertices labeled 0 and 1 is balanced. Each copy of $K_{1} \odot G$ has an extra edge of weight 0 . Therefore, when all the copies of $K_{1} \odot G$ are taken together, we have $m$ extra edges of weight 0 ; since the $n$ edges of $H$ have weight 1 and $|m-n| \leq 1$, the resulting labeling of $H \odot G$ is cordial.

In Figure 5 we show the case where $H \cong K_{2,3}$ and $G \cong P_{4}$.

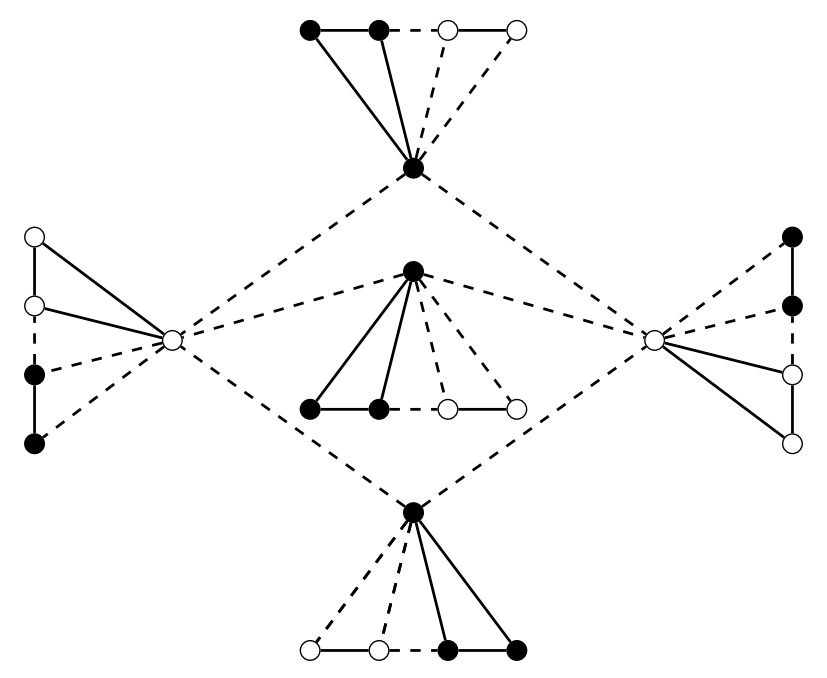

Figure 5. Cordial labeling of $K_{2,3} \odot P_{4}$

\section{Replicated Cordial Graphs}

In this section we consider two families of cordial graphs obtained using multiple copies of some cordial graphs. 
A circulant graph, denoted $\operatorname{Circ}(n ; X)$, is defined for a positive integer $n$ and a subset $X$ of integers $1,2, \ldots,\left\lfloor\frac{n}{2}\right\rfloor$, called the connections. The vertex set is $\mathbb{Z}_{n}$, the integers modulo $n$. There is an edge joining two vertices $j$ and $k$ if and only if the difference $|j-k|$ is in the set $X$. A circulant graph is a special case of a Cayley graph; circulant graphs are vertex transitive [7].

Theorem 3.1. If $n \geq 6$ is even and $X=\{1, k\}$, where $k$ is an even number, $k \leq \frac{n-2}{2}$, then $G=\operatorname{Circ}(n ; X)$ is cordial.

Proof. In this case, $G$ is a quartic graph of size $2 n$. For every $i \in \mathbb{Z}_{n}$, we define $f(i)=0$ if $i$ is even and $f(i)=1$ if $i$ is odd. Thus, the edges of the form $\{i, i+1\}$ have weight 1 . Since $k$ is an even number, the edges of the form $\{i, i \pm k\}$ have weight 0 . Then, $v_{f}(0)=v_{f}(1)=\frac{n}{2}$ and $e_{f}(0)=e_{f}(1)=n$. Therefore, $f$ is a cordial labeling of $G$.

In Figure 6 we show cordial labelings of the 3 circulant graphs with $n=16$, that is, $\operatorname{Circ}(16 ;\{1,2\})$, $\operatorname{Circ}(16 ;\{1,4\})$, and $\operatorname{Circ}(16 ;\{1,6\})$.

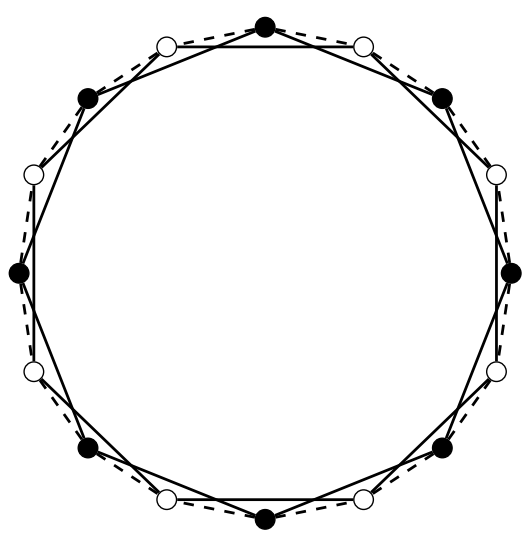

$\operatorname{Circ}(16 ;\{1,2\})$

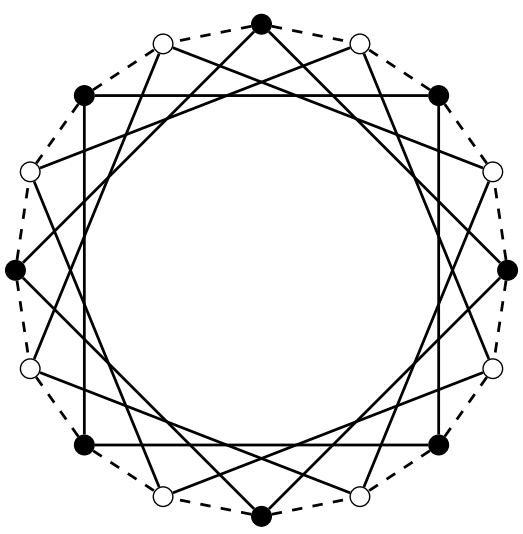

$\operatorname{Circ}(16 ;\{1,4\})$

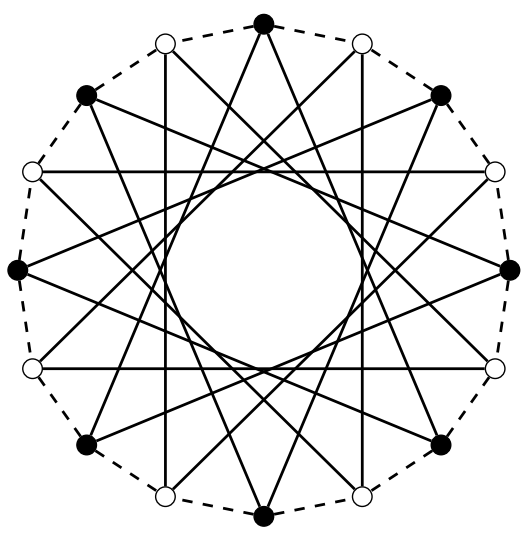

$\operatorname{Circ}(16 ;\{1,6\})$

Figure 6. Cordial labelings of circulant graphs

Using this result, the following natural generalization can be proven.

Corollary 3.1. If $n \geq 8$ is even and $X=\left\{g_{1}, g_{2}, \ldots, g_{t}, k_{1}, k_{2}, \ldots, k_{t}\right\}$, where every $g_{i}$ is $a$ generator of $\mathbb{Z}_{n}$ and every $k_{i}$ is an even number such that $k_{i} \leq \frac{n-2}{2}$, then $\operatorname{Circ}(n ; X)$ is cordial.

In Figure 7 we show the case where $n=16$ and $X=\{1,7,2,4\}$.

Let $G$ be a graph, for any positive integer $k$, the $k$-splitting graph of $G$, denote by $S^{k}(G)$, is obtained from $G$ by adding for each vertex $v$ of $G, k$ new vertices $v_{1}, v_{2}, \ldots, v_{k}$ so that every $v_{i}$ is adjacent to every vertex that is adjacent to $v$. If $G$ is a $(m, n)$-graph, then $S^{k}(G)$ has order $m(k+1)$ and size $n(2 k+1)$. 


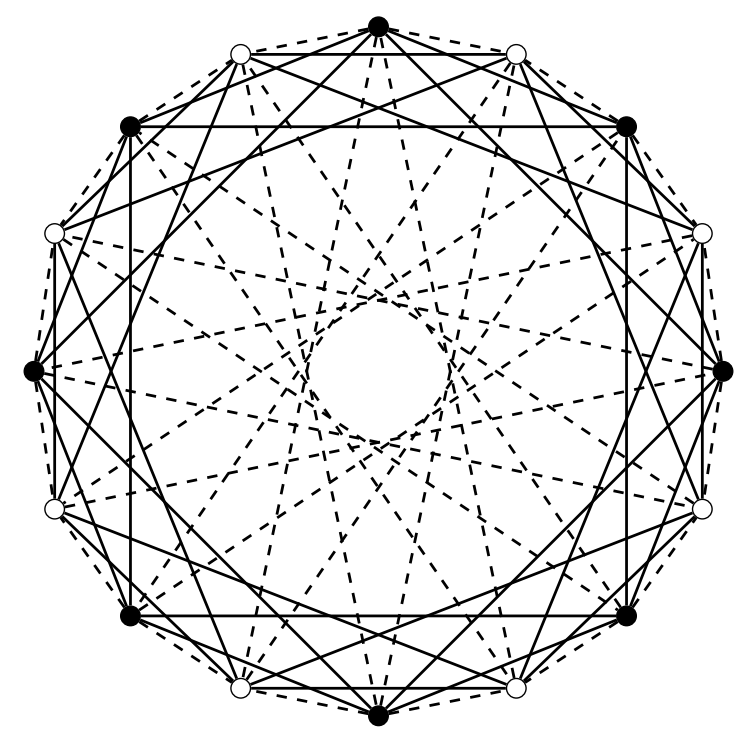

Figure 7. Cordial labeling of $\operatorname{Circ}(16 ;\{1,7,2,4\})$

It is known that the splitting graphs $S^{1}(G)$ are cordial when $G$ is the path $P_{n}$, the cycle $C_{n}$, the complete bipartite graph $K_{m, n}$, the wheel $W_{n}, n K_{2}$, and the bistar $B_{n, n}[9,15]$. In the next theorem we prove the cordiality of a broader family of splitting graphs. By $N(v)$ we understand the neighborhood of the vertex $v$.

Theorem 3.2. If $G$ is a cordial graph of even size, then the splitting graph $S^{k}(G)$ is cordial for every positive integer $k$.

Proof. Let $G$ be a cordial $(m, n)$ graph of even size and let $V(G)=\left\{v_{1}^{0}, v_{2}^{0}, \ldots, v_{m}^{0}\right\}$, consequently $V\left(S^{k}(G)\right)=\left\{v_{i}^{j}: 1 \leq i \leq m\right.$ and $\left.0 \leq j \leq k\right\}$. Suppose that $g$ is a cordial labeling of $G$ such that $v_{g}(0)-v_{g}(1) \geq 0$. We analyze two cases that depend on the parity of $m$.

Case 1: When $m$ is even.

Let $f$ be a binary labeling of $S^{k}(G)$ ) defined as $f\left(v_{i}^{j}\right)=g\left(v_{i}^{0}\right)$ for every $1 \leq i \leq m$ and $0 \leq j \leq k$. Since $m$ is even and $v_{g}(0)=v_{g}(1)$, we may conclude that $v_{f}(0)=v_{f}(1)$. In addition, because $N\left(v_{i}^{0}\right)=N\left(v_{i}^{j}\right)$, for each $1 \leq j \leq k$, the number of edges of weight 0 incident to $v_{i}^{0}$ is the same as the number of edges of weight 0 incident to $v_{i}^{j}$. Thus, $e_{f}(0)=\frac{n}{2}(2 k+1)$ and $v_{f}(0)=\frac{m}{2}(k+1)$. Therefore, $f$ is a cordial labeling of $S^{k}(G)$ ).

Case 2: When $m$ is odd.

Recall that in this case $v_{g}(0)-v_{g}(1)=1$. Without loss of generality we assume that $g\left(v_{1}^{0}\right)=0$. Let $f$ be a binary labeling of $S^{k}(G)$ defined, for every $2 \leq i \leq m$ and $0 \leq j \leq k$, as $f\left(v_{i}^{j}\right)=g\left(v_{i}^{0}\right)$, and for $i=1$ as $f\left(v_{1}^{j}\right)=0$ when $j \leq\left\lfloor\frac{k}{2}\right\rfloor$ and $f\left(v_{1}^{j}\right)=1$ when $j>\left\lfloor\frac{k}{2}\right\rfloor$. Thus, $v_{f}(0)=v_{f}(1)$ when $k$ is even and $v_{f}(0)=v_{f}(1)+1$ when $k$ is odd. Given that for every $i \geq 2$, the labeling of $v_{i}^{j}$ is the same that in Case 1, which is perfectly balanced, we just need to analyze the weight distribution of the edges incident to $v_{1}^{j}$. When $k$ is even, the edges incident to $v_{1}^{j}$, with $1 \leq j \leq \frac{k}{2}$, have a weight different than the edges $v_{1}^{\frac{k}{2}+j}$; since the edges of $G$ are totally balanced, so are the 
edges of $S^{k}(G)$. When $k$ is odd, the edges incident to $v_{1}^{j}$, for $0 \leq j \leq\left\lfloor\frac{k}{2}\right\rfloor$ have a weight different than the edges $v_{1}^{\left\lceil\frac{k}{2}\right\rceil+j}$, hence the weights of these edges are completely balanced and $f$ is a cordial labeling of $S^{k}(G)$.

As a consequence of this result we get that the splittings of any of the following graphs is cordial:

- A tree of even size.

- The complete bipartite graph, $K_{n, m}$, when the size is even.

- The wheel, $W_{n}$, for $n \equiv 0,1,2(\bmod 4)$.

- The cycle, $C_{n}$, for $n \equiv 0(\bmod 4)$.

- The $n$-sided prism, when $n \equiv 0(\bmod 4)$.

- The corona, $U \odot m K_{1}$, where $m$ is odd and $U$ is any unicyclic graph.

- The corona, $K_{1} \odot G$, where $G$ has odd size.

- The circulant graphs described before.

In Figure 8 we show an example of this construction where $G$ is the ladder $P_{4} \times P_{2}$.

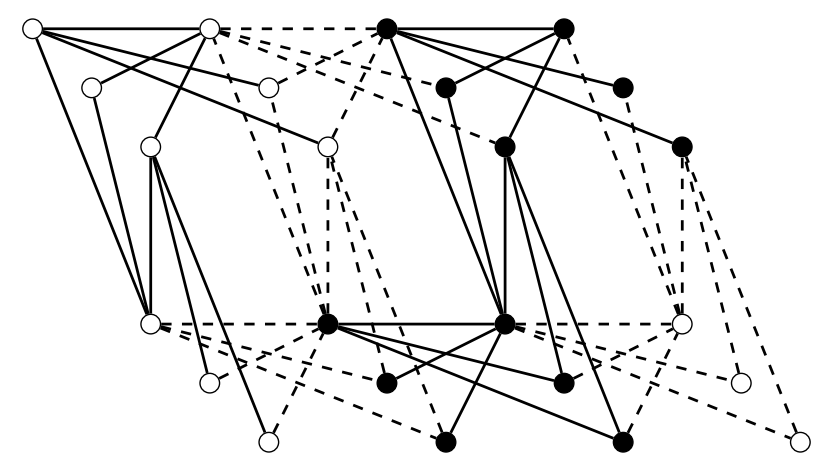

Figure 8. Cordial labeling of the splitting graph $S^{2}\left(P_{4} \times P_{2}\right)$

\section{Cordial Super Subdivision Graphs}

Sethuraman and Selvaraju [13] define a graph $H$ to be a super subdivision of a graph $G$, if every edge $u v$ of $G$ is replaced by $K_{2, m}$ ( $m$ may vary for each edge) by identifying $u$ and $v$ with the two vertices in $K_{2, m}$ that form the partite set with exactly two elements. Some authors have studied the cordiality of certain super subdivisions of graphs, among the graphs studied we can mention trees, 
complete bipartite graphs, and grids. The interested reader can find a detailed account of these results in Gallian' survey [6].

In [14], Sethuraman and Selvaraju proved that when every edge of a graph $G$ is super subdivided using graphs of the form $K_{2,2 m}$, the resulting graph is cordial. The following results are devoted to cordial labelings of super subdivisions of graphs using $K_{2,2 m+1}$ instead of $K_{2,2 m}$.

Lemma 4.1. The complete bipartite graph $K_{2,2 m+1}$ has a binary labeling $f_{1}$ such that $v_{f_{1}}(0)-$ $v_{f_{1}}(1)=3$ and $e_{f_{1}}(0)-e_{f_{1}}(1)=2$.

Proof. Let $A=\left\{u_{1}, u_{2}\right\}$ and $B=\left\{v_{1}, v_{2}, \ldots, v_{2 m+1}\right\}$ be the two partite sets of $V\left(K_{2,2 m+1}\right)$. Let $f_{1}$ be the binary labeling of $K_{2,2 m+1}$ defined as $f_{1}\left(u_{1}\right)=f_{1}\left(u_{2}\right)=0, f_{1}\left(v_{i}\right)=0$ if $1 \leq i \leq m+1$, and $f_{1}\left(v_{i}\right)=1$ if $m+2 \leq i \leq 2 m+1$. Thus, for every $1 \leq i \leq m+1$, the edges $u_{1} v_{i}$ and $u_{2} v_{i}$ have weight 0 , and for every $m+2 \leq i \leq 2 m+1$, the edges $u_{1} v_{i}$ and $u_{2} v_{i}$ have weight 1 . Therefore, $v_{f_{1}}(0)=m+3, v_{f_{1}}(1)=m, e_{f_{1}}(0)=2 m+2$, and $e_{f_{1}}(1)=2 m$.

Lemma 4.2. The complete bipartite graph $K_{2,2 m+1}$ has a binary labeling $f_{2}$ such that $v_{f_{2}}(0)-$ $v_{f_{2}}(1)=1$ and $e_{f_{2}}(0)-e_{f_{2}}(1)=-2$.

Proof. Let $A$ and $B$ be defined as in Lemma 4.1. Let $f_{2}$ be the binary labeling of $K_{2,2 m+1}$ defined as $f_{2}\left(u_{1}\right)=f_{2}\left(u_{2}\right)=0, f_{2}\left(v_{i}\right)=0$ when $1 \leq i \leq m$, and $f_{2}\left(v_{i}\right)=1$ when $m+1 \leq i \leq 2 m+1$. Thus, for every $1 \leq i \leq m$, the edges $u_{1} v_{i}$ and $u_{2} v_{i}$ have weight 0 ; for every $m+1 \leq i \leq 2 m+1$, the edges $u_{1} v_{i}$ and $u_{2} v_{i}$ have weight 1 . Therefore, $v_{f_{2}}(0)=m+2, v_{f_{2}}(1)=m+1, e_{f_{2}}(0)=2 m$, and $e_{f_{2}}(1)=2 m+2$.

Lemma 4.3. The complete bipartite graph $K_{2,2 m+1}$ has a binary labeling $f_{3}$ such that $v_{f_{3}}(0)-$ $v_{f_{3}}(1)=1$ and $e_{f_{3}}(0)-e_{f_{3}}(1)=0$.

Proof. Let $A$ and $B$ be defined as in Lemma 4.1. Let $f_{3}$ be the binary labeling of $K_{2,2 m+1}$ defined as $f_{3}\left(u_{1}\right)=0, f_{3}\left(u_{2}\right)=1, f_{3}\left(v_{i}\right)=0$ for $1 \leq i \leq m+1$, and $f_{3}\left(v_{i}\right)=1$ when $m+2 \leq i \leq 2 m+1$. Thus, for every $1 \leq i \leq m+1$, the edges $u_{1} v_{i}$ have weight 0 and the edges $u_{2} v_{i}$ have weight 1 ; for every $m+2 \leq i \leq 2 m+1$, the edges $u_{1} v_{i}$ have weight 1 and the edges $u_{2} v_{i}$ have weight 0 . Therefore, $v_{f_{3}}(0)=m+2, v_{f_{3}}(1)=m+1, e_{f_{3}}(0)=2 m+1$, and $e_{f_{3}}(1)=2 m+1$.

Let $\mathscr{E}$ be the family of all graphs for which there exists a binary labeling $f$ such that $\mid v_{f}(0)-$ $v_{f}(1) \mid \leq 1$ and $e_{f}(0)$ is even. The following graphs are members of $\mathscr{E}$ :

- Any balanced bipartite graph.

- If $G$ is a balanced bipartite graph of even order, then $n K_{1}+G \in \mathscr{E}$ for all $n \geq 1$.

- $K_{n}$ is a member of $\mathscr{E}$ for all $n \equiv 0,1,2(\bmod 4)$. Suppose that $n$ is even and $\frac{n}{2}$ vertices of $K_{n}$ are labeled 0 , then there are exactly $\frac{n(n-2)}{4}$ edges of weight 0 in $K_{n}$; since $n$ is even, $\frac{n(n-2)}{4}$ is also even. When $n \equiv 1(\bmod 4)$, exactly $\left\lceil\frac{n}{2}\right\rceil$ vertices of $K_{n}$ are labeled 0 , so the number of edges of weight 0 is given by $4 k^{2}$ where $n=4 k+1$. 
- The Cartesian product $P_{n} \times P_{m}$ when $n$ or $m$ is odd. This graph is bipartite, so by adding any even number of edges whose end-vertices have the same label, we produce a new graph with an even number of edges of weight 0 .

In the next theorem we prove that every super subdivision $H$ of a connected graph $G \in \mathscr{E}$ is cordial when the edges of $G$ are replaced by graphs of the form $K_{2,2 m+1}$.

Theorem 4.1. Every super subdivision $H$ of a connected graph $G \in \mathscr{E}$ is cordial when every edge of $G$ is replaced with a complete bipartite graph of the form $K_{2,2 m+1}$.

Proof. Suppose $G \in \mathscr{E}$, then there is a binary labeling $f$ of $G$ such that $\left|v_{f}(0)-v_{f}(1)\right| \leq 1$ and $e_{f}(0)$ is even. Let $E(G)=\left\{e_{1}, e_{2}, \ldots, e_{n}\right\}$, we denote by $K_{2,2 m_{i}+1}$ the complete bipartite graph used to super subdivide the edge $e_{i}$ of $G$.

Let $k$ be the number of edges of $G$ whose end-vertices are labeled 0 . Without loss of generality, we assume that both end-vertices of $e_{i}$ have label 0 when $1 \leq i \leq k$, and have label 1 when $k+1 \leq i \leq e_{f}(0)$, and that when $e_{f}(0)+1 \leq i \leq n$, the end-vertices of $e_{i}$ have different labels. We analyze two cases according to the parity of $k$. The labelings $f_{i}$ are those defined in the previous lemmas.

Case 1: When $k$ is even.

For every odd value of $i, 1 \leq i \leq k, K_{2,2 m_{i}+1}$ is labeled using $f_{1}$ and $K_{2,2 m_{i+1}+1}$ is labeled using $f_{2}$. In this way, the number of new vertices as well as the number of edges, labeled 0 and 1 , are totally balanced in the subgraph of $H$ induced by $K_{2,2 m_{i}+1}$ and $K_{2,2 m_{i+1}+1}$. For every odd value of $i, k+1 \leq i \leq e_{f}(0), K_{2,2 m_{i}+1}$ is labeled using $\bar{f}_{1}$ and $K_{2,2 m_{i+1}+1}$ is labeled using $\bar{f}_{2}$, and the result is the same. Finally, for every odd value of $i, e_{f}(0)+1 \leq i \leq n, K_{2,2 m_{i}+1}$ is labeled using $f_{3}$ and $K_{2,2 m_{i+1}+1}$ is labeled using $\bar{f}_{3}$. Recall that the labelings $f_{3}$ and $\bar{f}_{3}$ induce the same amount of edges of weight 0 than edges of weight 1 . If $e_{f}(1)$ is even, we have that the number of new vertices, in the subgraph of $H$ induced by $K_{2,2 m_{i}+1}$ and $K_{2,2 m_{i+1}+1}$, labeled 0 is the same as the number of new vertices labeled 1. Thus, the resulting labeling of $H$ is cordial. If $e_{f}(1)$ is odd, we proceed essentially in the same form, except that we select the labeling of $K_{2,2 m_{i}+1}$ based on the value of $v_{f}(0)-v_{f}(1)$. If $v_{f}(0)-v_{f}(1)=1$, we proceed as before, but if $v_{f}(0)-v_{f}(1)=-1$, $K_{2,2 m_{i}+1}$ is labeled using $\bar{f}_{2}$ and $K_{2,2 m_{i+1}+1}$ is labeled using $f_{2}$. Anyway, the final labeling of $H$ induces the same number of edges of weight 0 and 1, using the same number of vertices labeled 0 and vertices labeled 1 when $v_{f}(0)-v_{f}(1)=1$, or one more vertex labeled 1 . So, $H$ is cordial.

Case 2: When $k$ is odd.

We proceed as before, that is, for every odd value of $i, 1 \leq i \leq k, K_{2,2 m_{i}+1}$ is labeled using $f_{1}$ and $K_{2,2 m_{i+1}+1}$ is labeled using $f_{2}$. Up to this point, we have one more new vertex labeled 0 and two more edges of weight 0 . In order to compensate this disparity, we apply the labeling $\bar{f}_{2}$ to $K_{2,2 m_{k}+1}$. In this way, we have now, the same number of edges of weight 0 than edges of weight 1; however we have two more vertices labeled 0 than vertices labeled 1; this is going to be fixed when we super subdivide the edges of weight 1 of $G$. For every odd value of $i, k+2 \leq i \leq e_{f}(0)$, $K_{2,2 m_{i}+1}$ is labeled using $\bar{f}_{1}$ and $K_{2,2 m_{i+1}+1}$ is labeled using $\bar{f}_{2}$. Thus, when all the edges of weight 0 in $G$ have been super subdivided, we get that the new edges are totally balanced in terms of their weights and that there are two more new vertices with label 0 . Since $G$ is connected, $G$ has at least one edge of weight 1 . We call this edge $e_{e_{f}(0)+1}$, and $K_{2,2 m_{e_{f}(0)+1}}$ is labeled using $\bar{f}_{3}$. In this 
way, the edges are balanced and there is one more vertex labeled 0 than vertices labeled 1 . For every even value of $i, e_{f}(0)+2 \leq i \leq n, K_{2,2 m_{i}+1}$ is labeled using $\bar{f}_{3}$ and $K_{2,2 m_{i+1}+1}$ is labeled using $f_{3}$. In this way we have the same number of edges of weight 0 than edges of weight 1 . If $e_{f}(0)-e_{f}(1)$ is even, the number of vertices labeled 0 in $H$ equals the number of vertices labeled 1 ; if $e_{f}(0)-e_{f}(1)$ is odd, the number of vertices labeled 1 is one unit larger than the number of vertices labeled 0 . Therefore, the resulting labeling of $H$ is cordial.

In Figure 9 we show an example of a cordial super subdivision of the graph $C_{6}$.

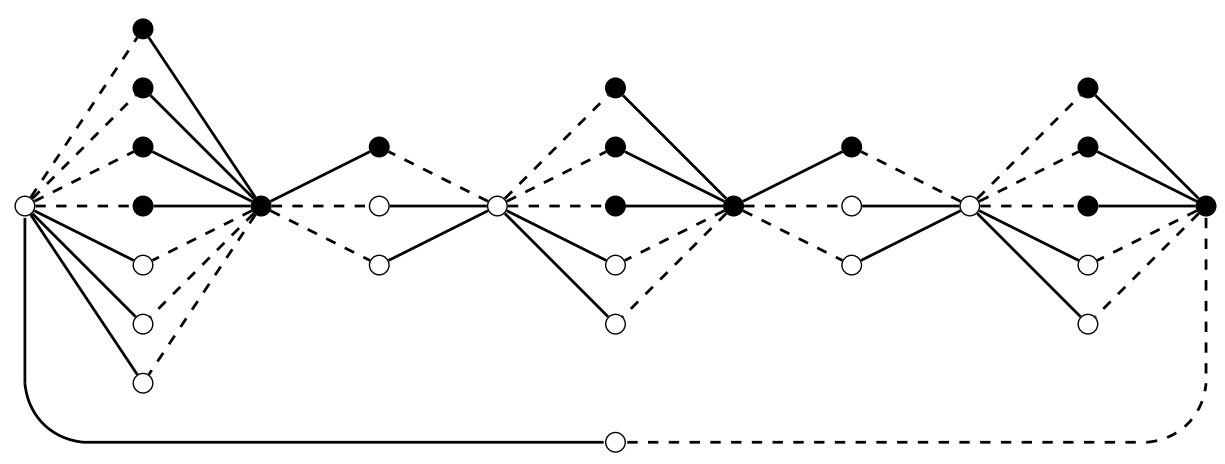

Figure 9. A cordial super subdivision of $C_{6}$

The following corollary is obtained by combining Theorem 16 and the result of Sethuraman and Selvaraju [14].

Corollary 4.1. If $G \in \mathscr{E}$, then every super subdivision $H$ of $G$ is cordial when every edge of $G$ is replaced with a complete bipartite graph of the form $K_{2,2 m+1}$ or $K_{2,2 m}$.

Remark 4.1. By using $m=1$ in the previous theorem, we have that the subdivision of all the edges of $G \in \mathscr{E}$ produces a cordial graph homeomorphic to $G$. The following question raises naturally. If $H$ is homeomorphic to $G \in \mathscr{E}$, is $H$ cordial?

Corollary 4.2. If $n \not \equiv 3(\bmod 4)$, then the graph $H$ obtained by subdividing every edge of $K_{n}$ is cordial.

Proof. Suppose that $n \equiv 3(\bmod 4)$, then $n=4 k+3$, for some integer $k$. The size of the subdivision $H$ of $K_{n}$ is $16 k^{2}+20 k+6 \equiv 2(\bmod 4)$. Since $K_{n}$ is Eulerian, $H$ is also Eulerian and has size congruent to $2(\bmod 4)$, therefore is not cordial.

Suppose now that $n \not \equiv 3(\bmod 4)$. The vertices of $K_{n}$ are separated in two sets, one with $\left\lfloor\frac{n}{2}\right\rfloor$ vertices labeled 1 by $f$, the other vertices labeled 0 by $f$. Thus, $v_{f}(0)-v_{f}(1) \leq 1$. When $n \equiv 0(\bmod 4), n=4 k$ for some positive integer $k$. Here every set induces $k(2 k-1)$ edges of weight 0 . Hence, $K_{n}$ has an even number of edges of weight 0 . Therefore $H$ is cordial. When $n \equiv 1(\bmod 4), n=4 k+1$ for some positive integer $k$. The first set induces $k(2 k-1)$ edges of weight 0 while the second set induces $k(2 k+1)$ edges of weight 0 . Then, $K_{n}$ has $4 k^{2}$ edges of 
weight 0 , which implies that $H$ is cordial. Finally, when $n \equiv 2(\bmod 4), n=4 k+2$ for some positive integer $k$. Now, each set induces $k(2 k+1)$ edges of weight 0 , and $K_{n}$ has an even number of edges of weight 0 . Therefore $H$ is cordial.

In Figure 10 we show a cordial subdivision of $K_{5}$ constructed on the binary labeling of $K_{5}$ exhibited on the left.
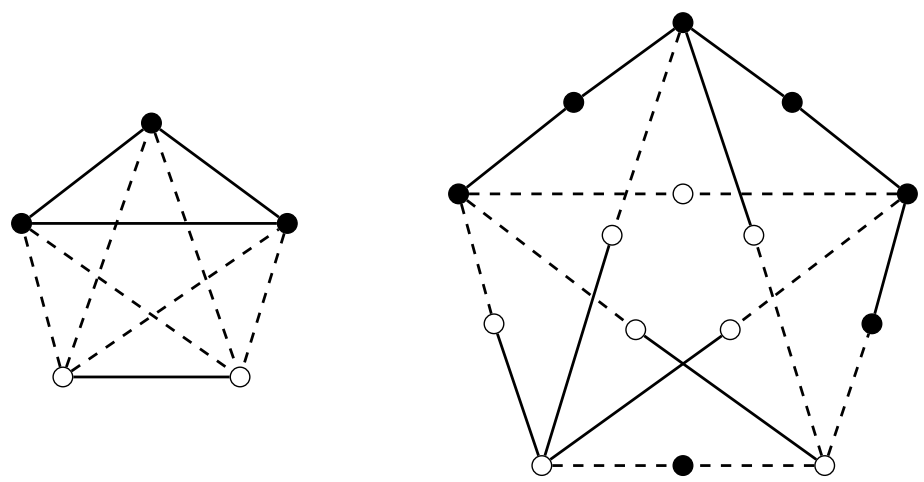

Figure 10. Cordial labeling of a subdivision of $K_{5}$

\section{Cordial Join Graphs}

In this section we study the cordiality of the join of two cordial graphs. Let $g$ and $h$ be cordial labelings of $G$ and $H$, respectively. Let $F=G+H$, thus $V(F)=V(G) \cup V(H)$ and $E(F)=$ $E(G) \cup E(H) \cup\{u v: u \in V(G), v \in V(G)\}$. Consider the binary labeling $f$ of $V(F)$ defined by

$$
f(w)= \begin{cases}g(w) & \text { if } w \in V(G), \\ h(w) & \text { if } w \in V(H) .\end{cases}
$$

We assume that one of the graphs, $G$ or $H$, has even order. Without loss of generality, suppose that $v_{g}(0)=v_{g}(1)=k$. If $v_{h}(0)=v_{h}(1)=l$, then there are $4 k l$ edges of the form $u v$ in $F$, with $u \in V(G)$ and $v \in V(H)$, and exactly $2 k l$ of them have weight 0 under $f$. If $v_{h}(0)=l$ and $v_{h}(1)=l+1$ or $v_{h}(0)=l+1$ and $v_{h}(1)=l$, then there are $4 k l+2 k$ edges of the form $u v$ in $F$, and exactly half of them have weight 0 under $f$. Therefore, to study the cordiality of $F$ we just need to analyze the values $e_{g}(i)$ and $e_{h}(i)$ for $i \in\{0,1\}$, because $\left|v_{f}(0)-v_{f}(1)\right| \leq 1$. Thus, $f$ is a cordial labeling of $F$ when $e_{g}(0)=e_{g}(1)$, or $e_{g}(0)=e_{g}(1)+1$ and $e_{h}(0) \neq e_{g}(1)+1$, or $e_{g}(0)+1=e_{g}(1)$ and $e_{h}(0)+1 \neq e_{g}(1)$.

Suppose now that both, $G$ and $H$, have odd order. If $v_{g}(0)=k+1$ and $v_{g}(1)=k$, then $v_{h}(0)=l$ and $v_{h}(1)=l+1$. The number of edges of the form $u v$ in $F$, is given by $4 k l+2 k+2 l+1$, and exactly $2 k l+k+l$ of them have weight 0 under $f$, that is, there is one more edge of weight 1 among the edges of the form $u v$.

Similarly, to determine whether $f$ is cordial, we just need to analyze the values of $e_{g}(i)$ and $e_{h}(i)$, for $i \in\{0,1\}$, because now $v_{f}(0)=v_{f}(1)$. Hence $f$ is always cordial, except when: 
- $e_{g}(0)=e_{g}(1)$ and $e_{h}(0)+1=e_{h}(1)$, or

- $e_{g}(0)+1=e_{g}(1)$ and $e_{h}(0)=e_{h}(1)$ or $e_{h}(0)+1=e_{h}(1)$.

Thus, we have proven the following general result about the cordiality of the join of two cordial graphs.

Theorem 5.1. The join of two cordial graphs, $G$ and $H$, is cordial if any of the following holds:

1. One of $G$ and $H$ has even order and even size.

2. Both $G$ and $H$ have odd order and even size.

3. Both $G$ and $H$ have odd order, odd size, and the dominating weight in both graphs is not 1 .

4. G has even order, odd size, and the dominating weight on both graphs is not the same.

5. Both $G$ and $H$ have odd order, but only one has odd size, and the dominating weight is 0 .

\section{Cordial One-Point Union Graphs}

Let $G$ be a graph and $v \in V(G)$, the one-point union of $t$ copies of $G$, denoted by $G^{(t)}$, is the graph obtained from $t$ copies of $G$ that share the vertex $v$. Shee and Ho [8] investigated the cordiality of several graphs of this form, such as $C_{m}^{(t)}, K_{m}^{(t)}, W_{m}^{(t)}, F_{m}^{(t)}$, and $F l_{m}^{(t)}$. Andar et al. [1], also investigated the one-point union of graphs, where $G$ is a helm, closed helm, flower, gear, sunflower, or a ply. See [6] for more details about these contributions.

The following results show cordial labelings of graphs of the form $G^{(t)}$, where $G$ is any cordial graph of odd order and even size, or $G$ is a special type of cordial graph of odd order and odd size. Thus, some of the results mentioned above are just specific cases of these two theorems.

Among the families of cordial graphs of odd order and even size we have:

- $C_{3}^{(4 t)}[3]$.

- $K_{m, n}$ when $m$ is odd and $n$ is even [2].

- Any cactus of size $n \equiv 0(\bmod 4)$ whose blocks are cycles [9].

- $m K_{1}+G$, where $G$ is a cordial graph of even order and $m$ is an odd positive integer.

- $G \odot 2 n K_{1}$, where $G$ is a cordial graph of odd order and even size.

Theorem 6.1. If $G$ is a cordial graph of odd order and even size, then there is a one-point union of t copies of $G$ that is cordial.

Proof. Without loss of generality we assume that $v_{f}(0)-v_{f}(1)=1$. Let $v \in V(G)$ such that $f(v)=0$. Identifying the corresponding vertices $v$ of each copy of $G$ we have a one-point union graph with a binary labeling $g$. We claim that $g$ is a cordial labeling. In fact, in every copy of $G-v, v_{g}(0)=v_{g}(1)$, so in the one-point union graph, $v_{g}(0)-v_{g}(1)=1$; since $e_{f}(0)-e_{f}(1)=0$ and there are no new edges, $e_{g}(0)-e_{g}(1)=0$. Therefore, the labeling $g$ of $G^{(t)}$ is cordial. 

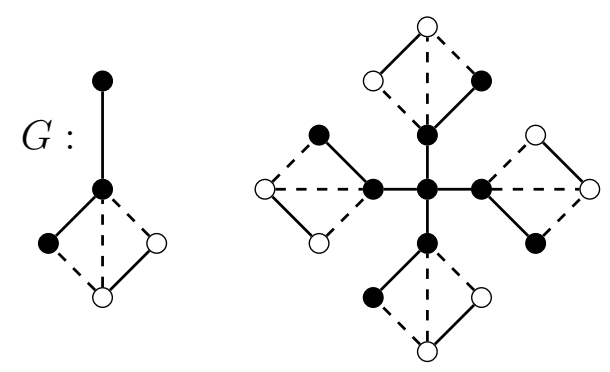
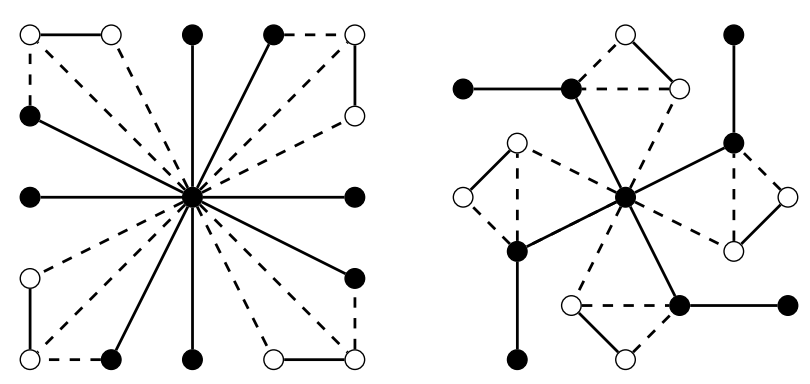

Figure 11. Cordial one-point union graphs obtained from a cordial base graph $G$

In Figure 11 we show three examples of this one-point union that use the same graph $G$.

Let $\mathscr{S}$ be the class of all cordial graphs of odd order and odd size such that for any $G \in \mathscr{S}$ there exist two cordial labelings, $f$ and $g$, such that $e_{f}(0)-e_{f}(1)=1, e_{g}(0)-e_{g}(1)=-1$, and a vertex $v \in V(G)$ for which $f(v)=g(v)=0$.

The following two families of graphs correspond to members of $\mathscr{S}$.

- Let $n \geq 3$ be an odd integer and let $G$ be the graph obtained from $K_{2, n}$ by adding an edge connecting any pair of vertices in the largest stable set of $K_{2, n}$; thus, this graph has odd order and odd size. We claim that $G \in \mathscr{S}$. In fact, if $V(G)=\left\{u_{1}, u_{2}, v_{1}, v_{2}, \ldots, v_{n}\right\}$ and $E(G)=\left\{u_{i} v_{j}: i=1,2\right.$ and $\left.j=1,2, \ldots, n\right\} \cup\left\{v_{n-1} v_{n}\right\}$, then the labeling $f$ of the vertices of $G$ defined by $f\left(u_{1}\right)=1, f\left(u_{2}\right)=0$, and

$$
f\left(v_{j}\right)= \begin{cases}1 & \text { if } 1 \leq j \leq\left\lfloor\frac{n}{2}\right\rfloor, \\ 0 & \text { if }\left\lfloor\frac{n}{2}\right\rfloor<j \leq n,\end{cases}
$$

is cordial where $v_{f}(0)-v_{f}(1)=1$ and $e_{f}(0)-e_{f}(1)=1$ due to the fact that $f\left(v_{n-1}\right)=$ $f\left(v_{n}\right)=0$.

Consider now the labeling $g$ of $G$ defined by $g\left(u_{1}\right)=1, g\left(u_{2}\right)=0$, and

$$
g\left(v_{j}\right)= \begin{cases}1 & \text { if } j \text { is odd } \\ 0 & \text { if } j \text { is even } .\end{cases}
$$

This labeling is also cordial; note that $v_{g}(0)-v_{g}(1)=1$ and $e_{g}(0)-e_{g}(1)=-1$, because $g\left(v_{n-1}\right)=1$ and $g\left(v_{n}\right)=0$. When these labelings are restricted to $K_{2, n}$ they are the same as the cordial labeling of this graph introduced by Cahit in [2]. Note that essentially all vertices of $G$, except $v_{n-1}$, have the same label with both labelings.

- Let $C_{n}$ be a cycle of odd order where $n \geq 5$. Label the vertices of $C_{n}$ using the cordial labelings given in [2]. When $n \equiv 1(\bmod 4)$, let $G$ be the outerplanar graph obtained from $C_{n}$ by adding the edges $v_{1} v_{3}$ and $v_{1} v_{n-1}$, the induced labeling of this graph satisfies $e(0)-e(1)=$ -1 . Let $G^{\prime}$ be the outerplanar graph obtained from $C_{n}$ by adding the edges $v_{2} v_{4}$ and $v_{2} v_{n}$. Clearly, $G \cong G^{\prime}$ and the induced labeling of this graph satisfies $e(0)-e(1)=1$. So we have 
that $G \in \mathscr{S}$. When $n \equiv 3(\bmod 4)$, the graph $G$ is obtained, similarly, by adding the edges $v_{1} v_{4}$ and $v_{1} v_{n-2}$, while $G^{\prime}$ is obtained by adding the edges $v_{2} v_{5}$ and $v_{2} v_{n-1}$.

We claim that there are cordial one-point union graphs obtained using graphs from $\mathscr{S}$.

Theorem 6.2. If $G \in \mathscr{S}$, then there is a one-point union of $G$ that is cordial.

Proof. Without loss of generality, we assume that $v_{f}(0)-v_{f}(1)=1$ and $v_{g}(0)-v_{g}(1)=1$. Consider $t$ copies of $G$, where $\left\lceil\frac{t}{2}\right\rceil$ of these copies are labeled using $f$ and $\left\lfloor\frac{t}{2}\right\rfloor$ are labeled using $g$. Suppose that $v \in V(G)$ has label 0 under both labelings, then we identify the vertex $v$ of each copy of $G$ to produce the one-point union graph. Let $h$ denote the binary labeling of the resulting onepoint union graph. Thus, $v_{h}(0)-v_{h}(1)=1$ and $e_{h}(0)-e_{h}(1)=1$ if $t$ is odd and $e_{h}(0)-e_{h}(1)=0$ when $t$ is even. Therefore, $h$ is cordial regardless the parity of $t$.

In Figure 12, we show an example of this one-point union.

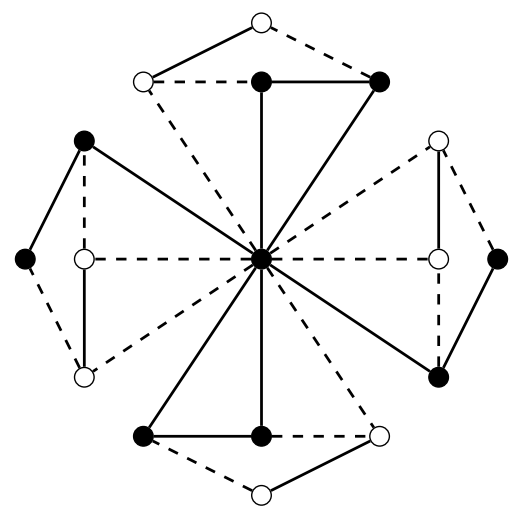

Figure 12. Cordial labeling of a one-point union graph

\section{References}

[1] M. Andar, S. Boxwala, and N. Limaye, On the cordiality of corona graphs, Ars Combin., 78 (2006), 179-199.

[2] I. Cahit, Cordial graphs: a weaker version of graceful and harmonious graphs, Ars Combin., 23 (1987), 201-207.

[3] I. Cahit, On cordial and 3-equitable labellings of graphs, Util. Math., 37 (1990), 189-198.

[4] G. Chartrand and L. Lesniak, Graphs \& Digraphs 2nd ed., Wadsworth \& Brooks/Cole (1986).

[5] G. M. Du, Cordiality of complete $k$-partite graphs and some special graphs, Neimenggu Shida Xuebao Ziran Kexue Hanwen Ban, (1997), 9-12. 
[6] J. A. Gallian, A dynamic survey of graph labeling, Electronic J. Combin., 23 (2020) \#DS6.

[7] J. L. Gross, J. Yellen, and P. Zhang, Handbook of Graph Theory 2nd ed. CRC Press (2014).

[8] Y. S. Ho, S. M. Lee, and S. C. Shee, Cordial labellings of the Cartesian product and composition of graphs, Ars Combin., 29 (1990), 169-180.

[9] W.W. Kirchherr, On the cordiality of some specific graphs, Ars Combin., 31 (1991), 127-138.

[10] Z. Liu and B. Zhu, A necessary and sufficient condition for a 3-regular graph to be cordial, Ars Combin., 84 (2007), 225-230.

[11] R. C. Read and R. Wilson, An Atlas of Graphs, Oxford University Press (1998).

[12] M. A. Seoud and A. E. I. Abdel Maqsoud, On cordial and balanced labelings of graphs, $J$. Egyptian Math. Soc., 7 (1999), 127-135.

[13] G. Sethuraman and P. Selvaraju, Gracefulness of arbitrary supersubdivisions of graphs, Indian J. Pure Appl. Math., 32 (2001), 1059-1064.

[14] G. Sethuraman and P. Selvaraju, Decompositions of complete graphs and complete bipartite graphs into isomorphic supersubdivision graphs, Discrete Math., 260 (2003), 137-149.

[15] M. Z. Youssef, On Skolem-graceful and cordial graphs, Ars Combin., 78 (2006), 167-177.

[16] M. Z. Youssef, Graph operations and cordiality, Ars Combin., 97 (2010), 161-174. 